\title{
Experiencias de Educación Técnica Rural en escuelas públicas del Norte Argentino: el caso de Salta
}

\author{
Marcelo Gastón Jorge Navarro \\ mjorgenavarro@conicet.gov.ar
}

Talía Gutiérrez ${ }^{2}$

tgutierrez@unq.edu.ar

\section{Resumen}

Desde el siglo XIX, en Argentina surgieron diferentes propuestas de enseñanza orientadas a la formación técnica en el ámbito rural. Con distintos matices que va desde orientaciones en ganadería hasta trabajo agropecuario regional, las diversas formas que adquirió esta modalidad de educación fue variando y mutando, vincularmente no solo con los contextos sociopolíticos y los momentos históricos en el que se desarrollaban, sino también, en concordancia con las diferentes influencias de pensamiento que otorgan particularidades a estas propuestas. El siguiente artículo desanda alguna de esas tendencias a lo largo de su historia, pero haciendo hincapié en las propuestas ejecutadas en la provincia de Salta, al norte de Argentina, particularmente en las escuelas del Programa de Expansión y Mejoramiento de la Educación Técnica Agropecuaria (EMETA) intentando brindar un panorama general de las distintas acciones que se orientaron a la educación técnica en escuelas rurales medias.

Palabras claves: educación técnica; mejoramiento agropecuario; escuelas EMETA.

\footnotetext{
1 Doutorando em Ciências Sociais e Humanidades, Universidade Nacional de Quilmes, Argentina. 2 Doutora em História pela Universidad Nacional de La Plata (2005). Professora da Universidad Nacional de Quilmes da Universidad Nacional de La Plata.
} 


\title{
Experiences Technical Education in public schools Rural Northern Argentina: the case of Salta
}

\begin{abstract}
Since the nineteenth century, in Argentina emerged different teaching approach oriented technical training in rural areas. With different shades ranging from guidance in regional livestock to agricultural work, various forms which acquired this mode of education was changing and mutating, related not only sociopolitical contexts and historical moments in which they were developed, but also, in accordance with the different influences that give special thought to these proposals.

This article retraces some of these trends over its history, but with emphasis on the proposals implemented in the province of Salta, in northern Argentina, particularly in schools Expansion Program and Technical Education Improvement Agricultural (EMETA) trying to provide an overview of the various actions that were directed to the technical education in rural middle schools.
\end{abstract}

Keywords: technical education; agricultural improvement; Schools EMETA.

\section{Primeras experiencias de enseñanza agrícola en Argentina}

Desde el siglo XIX, en Argentina surgieron diferentes propuestas de enseñanza orientadas a la formación técnica en el ámbito rural. Desde las propuestas de Juan Manuel Belgrano, Bernardino Rivadavia y Juan Vieytes, pasando por las misiones monotecnicas y escuelas agrícolas de finales de 1950, hasta el programa de escuelas EMETA a finales del siglo XX. Con distintos matices que va desde orientaciones en ganadería hasta trabajo agropecuario regional, las diversas formas que adquirió esta modalidad de educación fue variando y mutando, vincularmente no solo con los contextos sociopolíticos y los momentos históricos en el que se desarrollaban, sino también, en concordancia con las diferentes influencias de pensamiento que otorgan particularidades a estas propuestas. 
Si bien el desarrollo y consolidación de la educación técnica rural va a producirse a partir de la segunda mitad del siglo XX, las bases y orígenes de las propuestas sobre un tipo de educación vinculada al mundo rural, van a surgir en el siglo XIX.

La educación rural, principalmente en el nivel medio fue pensada desde las políticas estatales con una lógica progresista, que necesitaba orientar a determinadas clases hacia un trabajo manual, de índole agrícola y ganadera. En este sentido, la educación agrícola, se pensaba necesaria para la consolidación de un determinado orden social, a partir del cual se podría efectivizar un certero control social, que asegurase la incorporación de determinados valores y normas por parte de ciertos sujetos, pertenecientes a algunas clases sociales.

La enseñanza técnica rural en Argentina tiene como primer antecedente la "La escuela de agricultura y Jardín de Aclimatación" fundada por Bernardino Rivadavia en 1823 en la quinta de la Recoleta por decreto del 7 de Agosto, con un plan de estudio de 2 años. Esta escuela tenía como antecedente la propuesta de Manuel Belgrano, quien proyectó una Escuela Práctica de Agricultura, para impulsar el cultivo racional de las tierras y delineó un plan de escuela de Artes y Oficios, con el fin de expandir la vocación por el trabajo manual "Se trataba de promover a la reforma de las prácticas agrícolas mediante la capacitación técnica de los labradores" (SALVADORES, 1940, p. 6)

A partir de esta experiencia inicial, se sucederán muchas más propuestas de escuela. En 1870, se crean secciones de agricultura en los Colegios Nacionales de Salta, Tucumán y Mendoza, siendo la primera suprimida al poco tiempo de su inauguración, mientras que las dos últimas se conformaron posteriormente en las llamadas "quintas agronómicas" (AMADEO, 1916, p. 6). En el año 1871 se crean además los Departamentos de Minerías en los Colegios de San Juan y Catamarca por iniciativas de Domingo F. Sarmiento.

A ellas habría que sumarles las experiencias en las escuelas de Córdoba con la instrucción agrícola femenina en escuelas comunes, las escuelas nacionales de Agricultura de Casilda y de Córdoba, y la Escuela del Hogar Agrícola en Tandil (Bs As), entre muchas más. El 
sistema comprendía escuelas especiales de nivel superior y prácticas de nivel elemental.

En el caso de la provincia de Salta, se destaca fundación de la escuela práctica de agricultura de Puerta de Díaz en 1911, posteriormente denominada estación experimental (1929), y los cursos temporarios del hogar agrícola en el periodo 1918- 1919 en la localidad de Rosario de Lerma (GUTIÉRREZ, 2007).

A comienzos del siglo XX las escuelas rurales solo brindaban educación hasta segundo o tercer año de educación elemental. En el caso de la enseñanza agrícola, en las primeras décadas, circulaban algunas propuestas especializadas, que tomaban como base modelos implementados en el extrajeron, que se creía podrían aplicarse en nuestro país (GUTIÉRREZ, 2007, p. 79).

Estas propuestas estaban enmarcadas en la fuerte tendencia normalista de la época, que tenía fundamentos claramente positivistas, enarbolando banderas como la ciencia, para el progreso social. Uno de los representantes de esta tendencia, fue Víctor Mercante, quien veía en la educación agrícola un elemento de necesidad de desarrollo psico- físico importante para el desarrollo del sujeto. De esta forma se contribuía al desarrollo científico en el niño, mediante la observación de los fenómenos, y el trabajo manual que fomentaría el desarrollo moral (VERGARA, 1897).

Hacia la década de 1940, las migraciones internas, de las zonas rurales a las zonas urbanas se acrecentaron aún más, debido a los procesos de industrialización, y la creciente demanda de mano de obra en la insipiente industria nacional, ubicada alrededor de las grandes ciudades de la región pampeana, pero que no alcanzaba a provincias del NOA como Salta.

A partir de 1943, varias fueron las modificaciones socioeconómicas que tuvieron que ver con lo rural, entre ellos, destaca la sanción del “Estatuto del Peón” en octubre de 1944. El primer plan quinquenal sostenía la necesidad de ampliar el sistema educativo vigente. La ampliación contemplaba todos los niveles del sistema, con la idea de garantizar un mayor acceso por parte de población a la educación. Idea muy vinculada en el peronismo con la idea de la "Justicia social". 
Con la asunción del peronismo al poder, la formación técnica en el sistema educativo, se acrecentó. Y este crecimiento también abarcó la educación técnica en el ámbito rural. Las reformas aplicadas por el peronismo comprendían tanto el nivel primario con cursos de preaprendizaje y las misiones monotécnicas, como el nivel medio, con las escuelas-fábricas e industriales de la Nación, llegando inclusive al nivel superior con la creación de la Universidad Obrera Nacional, aunque estas últimas vinculadas al ámbito urbano.

En 1947 se crearon las "misiones monotécnicas y de extensión cultural" para varones y las "misiones de cultura doméstica" para las mujeres. Las misiones tenían el objetivo de atender a la población rural, constituyéndose en una oferta que abarcaba a jóvenes y adultos que hubieran terminado el cuarto grado de la educación primaria. Las misiones brindaban capacitación técnica en una especialidad, además de impartir los contenidos correspondientes al quinto y sexto grado de la escuela primaria.

Las misiones se proponían brindar conocimientos prácticos elementales, no reduciéndose a la capacitación en un oficio, sino fundamentándose en una amplia formación cultural. Además de retener a los jóvenes de pequeñas poblaciones rurales, y evitar la migración a las grandes urbes.

Las misiones estaban dirigidas a aquella población de alumnos del interior, que habían abandonado la educación primaria, o que hubiesen repetido grados. Los requerimientos para el ingreso eran: tener aprobado el cuarto grado o rendir un examen de competencias; tener cumplidos 14 años de edad; presentar solicitud de ingreso, aval de padre o tutor; $y$ certificado médico de aptitud física.

Quedaba establecido que en cada localidad, la misión tenía un carácter transitorio, estimado en dos años de permanencia. Sin embargo era común que la permanencia de la misión se extendiera más de lo estipulado. El departamento de Misiones Monotécnicas podía autorizar la permanencia de la Misión por otros dos años más.

En el periodo 1947-55, según los datos de la Secretaria de Educación de la Nación, funcionaron 70 misiones, en 123 localidades. En el caso de 
la provincia de Salta, funcionaron 2 misiones monotécnicas entre 1947 y 1955, las mismas funcionaban en las localidades de Metan y Rosario de Lerma.

\section{Experiencias de educación Agropecuarias en la segunda mitad del siglo XX: las escuelas EMETA}

Ya a mediados de siglo XX las propuestas sobre la educación agrotécnica habían alcanzado muchas variantes en los distintos niveles del sistema, y aunque se ponía el hincapié especialmente en la enseñanza media, no faltaron las propuestas y experiencias en el nivel primario y superior, especialmente en la formación docente.

Hacia 1960 Jorge Stagnaro en un trabajo presentado en las Jornadas Pedagógicas Panamericanas, realizadas en Buenos Aires, en Noviembre de 1960, sostenía que de todos los males que afecta a la Argentina, el más importante es el déficit educativo, especialmente en las zonas rurales.

En su descripción de las escuelas rurales, señala como características relevantes la constitución étnica de la población, las condiciones económicas de los mismos, las dificultades geográficas, y hasta factores vinculados a la asistencia social, la comunicación y a la cultura. Por ello, señala, que el maestro debe estar preparado para enfrentar tales desafíos, a partir de una sólida formación y una voluntad firme.

Según los datos estadísticos que presenta el informe de Stagnaro, la provincia de Salta tenía 460 escuelas en total de las cuales 65 eran urbanas, y 395 rurales. De estas últimas 253 tenían ciclo incompleto, 177 contaban con un solo maestro. Asistiendo a estas escuelas rurales 32.260 alumnos, y teniendo 1.148 maestros.

En la época existían varias instituciones en el país encargadas de formar docentes rurales, uno era el Instituto Superior de Educación Rural "Eduardo Olivera" en Tandil, creada en 1955 como "Eva Perón" y el otro, era el Instituto Superior del Hogar Agrícola “Ing. Agr. Dr. Tomas Amadeo" dependiente de la Secretaria de Estado de Agricultura y Ganadería de la Nación, en la ciudad de Bolívar, ambas en la provincia de Buenos Aires (GUTIÉRREZ, 2007). También estaban las “escuelas normales de adaptación regional" del Ministerio de Justicia e Instrucción 
Pública, y la escuela de maestros rurales Alberdi de Entre Ríos.

Ya ddurante la década de 1970 se produjo un fuerte proceso de expansión de los establecimientos de educación agraria. En este sentido, se dispuso de una variada oferta tanto pública como privada de educación técnica rural a nivel nacional que fue acompañada por emprendimientos provinciales que también crecieron en esa etapa, a partir aproximadamente de 1975.

En este contexto y desde 1980, con financiamiento del Banco Interamericano de Desarrollo, surge un programa destinado a la escuela media rural y pensado para la aplicación en distintas regiones del país. El mismo se denominaba Programa de Expansión y mejoramiento de la Educación Técnica Agropecuaria (E.M.E.T.A.) y comprendía un conjunto de subprogramas destinados al mejoramiento de la modalidad agropecuaria en el sistema educativo (LEGUIZAMÓN; JORGE NAVARRO, 2013) El mismo era definido como un programa que:

Comprende un conjunto de subprogramas desarrollados, en forma coordinada encaminados a lograr un mejor rendimiento del sistema educativo, así como una ampliación y mejoramiento de los servicios educacionales en el ámbito agropecuario. Para los efectos del Programa se entiende por sistema educativo el referido a la educación técnica agropecuaria (formal y no formal). (ARGENTINA, 1988, p. 7)

Este programa se constituyó en el marco de una serie de proyectos financiados por organismos internacionales para América Latina con el objetivo de desarrollar la productividad en zonas rurales mediante "la modernización agrícola" y teniendo como eje las políticas educativas. Fue así como el "Programa de Expansión y Mejoramiento de la Educación Técnica agropecuaria" financiado parcialmente por el Banco Interamericano de Desarrollo mediante los contratos de préstamos $\mathrm{N}^{\circ}$ 123/IC y 718/SF y en convenio con la Nación Argentina fueron tomando fuerza a finales de la década del ' 80 .

Su primer antecedente fue el Programa de Expansión y Mejoramiento de la Educación Rural (EMER) Programa que fuera ejecutado entre el Ministerio de Cultura y Educación de la Nación y el Banco Interamericano de Desarrollo (BID), aprobado por Decreto No1163 del 24 de marzo de 
1979 y que duró hasta adentrado los primeros años de la década de los 90.

EMER y EMETA eran programas que en un momento fueron contemporáneos con aplicación sobre el total del país, cuyo financiamiento dependía del Banco Interamericano de Desarrollo (BID) en un 50\% y el restante $50 \%$ estaba a cargo del estado argentino. Para el caso de EMETA se implementaron en total 25 proyectos con 25 Unidades Ejecutoras locales, en 22 provincias. Los convenios se firmaron en setiembre de 1985 (MARTÍNEZ GUARINO, 1994, p. 39).

La aplicación estaba a cargo de una Unidad Ejecutora Central (U.E.C.) del Ministerio de Educación de la Nación y se encargaba de coordinar el Programa a nivel nacional, mientras que en las provincias la Unidad Ejecutora Provincial (U.E.P.) era la encargada de la administración y ejecución del Proyecto.

El programa estructuraba estudios de base, desarrollo curricular, perfeccionamiento docente, becas a estudiantes de bajo recursos y cursos de extensión para la capacitación laboral en las comunidades donde se insertaban. Además de la construcción o refacción de escuelas para el funcionamiento del programa y el otorgamiento de equipamiento para el desarrollo de las actividades previstas.

En base a estos lineamientos fue que en el año 1988 la provincia de Salta comenzó su proceso de recolección de datos, búsqueda de información y elaboración de informes en distintos aspectos de las zonas seleccionadas por las propia provincia de acuerdo a las características que presentaban y acorde a estrategias de desarrollo local, concluyendo en lo que se transformó en los estudios de base para la implementación del programa EMETA.

Estos documentos diagnósticos de base eran estudios que se realizaron en las provincias previo a la aplicación del programa, su contenido resultaba central puesto que procuraba brindar una descripción de la realidad local (educación, salud, economía, principales producciones, principales problemáticas) a fin de que el mismo se viera reflejado en la propuesta final sobre la orientación productiva y el plan de estudios, como tal estos diagnósticos constituyen una fuente relevante de información sobre el tema. 


\section{Programa de Expansión y mejoramiento de la Educación Técnica Agropecuaria: antecedentes e informes diagnósticos en Metan y Yacuy.}

El 1 de septiembre de 1988 el Senado y la cámara de diputados de la Provincia de Salta sancionan la ley $\mathrm{N}^{\circ} 6527$ que ponen en funcionamiento y mantenimiento el programa de Expansión y Mejoramiento de la Educación Técnica Agropecuaria (EMETA), siendo ratificada esta ley por Decreto $N^{\circ} 2122$ del Poder Ejecutivo, el 29 de Septiembre del mismo año.

Convenio celebrado con el Ministerio de Educación y Justicia de la Nación, para acordar las normas y condiciones básicas para la ejecución, puesta en funcionamiento y mantenimiento de un Programa de Expansión y Mejoramiento de la Educación Técnica Agropecuaria (EMETA); y CONSIDERANDO: Que por el aludido convenio, la Nación toma a su cargo la transferencia, sin reintegro, de los recursos necesarios para la ejecución del conjunto de acciones que integran el programa en cuestión; Que en mérito a lo establecido en el artículo 14 - Inc. 13, de la Constitución de la Provincia, es facultad del Poder Ejecutivo la celebración de acuerdos como el presente, sujetos a la aprobación del Poder Ejecutivo. (ARGENTINA, 1987)

El decreto 2122 manifestaba que los recursos y el financiamiento para la ejecución del programa estaría a cargo del Estado Nacional, con la transferencia a la provincia de un monto equivalente a u\$s 4.049.628,92 en australes (moneda vigente en ese momento en Argentina a partir del Plan Austral de 1985), originados parcialmente por el contrato de préstamo celebrado entre la Nación y el Banco Interamericano de Desarrollo, y complementado con los aportes del Estado Nacional.

También estipulaba la creación de una Unidad Ejecutora Provincial (U.E.P.) facultada para ejecutar las acciones del programa, administrar y disponer de fondos, y celebrar convenios con la Unidad Ejecutora Central (U.E.C.). Es en este punto que solieron suscitarse controversias en las provincias donde se aplicó el Programa, con relación en especial a la disposición de los fondos, la elección de las unidades educativas a las que se dedicaron, etc.

El reglamento de ejecución del programa EMETA estipulaba u\$s 
133.400.000 equivalente en australes ${ }^{3}$ para el desarrollo de las acciones previstas. De ese monto total u\$s 78.500 .000 eran provenientes del préstamo $\mathrm{N}^{\circ} 718 / \mathrm{SF}-\mathrm{AR}$ y $\mathrm{N}^{\circ} 123 / \mathrm{IC}-\mathrm{AR}$ del B.I.D., y u\$s 54.900 .000 eran aportados íntegramente por la Nación Argentina, las que serían transferidas a las jurisdicciones en forma no reembolsables.

Previa ejecución del programa, era necesario la elaboración de una serie de estudios diagnósticos de factibilidad que dieran cuenta de las condiciones y viabilidades de aplicación en una determinada región. Fue así que la provincia de Salta realizó 2 estudios diagnósticos, de varias etapas cada uno, en las localidades de Yacuy en el departamento de Oran al norte provincial, y otro en la localidad de Metan (del departamento homónimo) al sur de Salta.

La elección de estas localidades para la realización de los estudios diagnósticos y posterior implementación del programa, respondía a las ubicaciones estratégicas de ambas zonas y a su potencial productivo.

La localidad de Yacuy con amplia influencia desde el límite norte de la frontera con Bolivia, hasta las localidades de Colonia Santa Rosa, Pichanal, Oran incluyendo las localidades ubicadas sobre la ruta 34 y la localidad de Coronel Cornejo. En cuanto a su producción destacaba la aptitud geográfica para el cultivo intensivo de bajo riesgo hacia el norte, y bajo cultivo extensivo hacia el sur.

En el caso de Metan, su ubicación estratégica al sur de la provincia, viejo paso obligado de transporte y comercio, presentaba una zona de influencia que incluía a Rosario de la Frontera, Anta y las poblaciones del Rio Piedra. Su potencial productivo estaba en la aptitud predominantemente agrícola, por la profundidad de sus suelos con buena disponibilidad de nutrientes y materia orgánica. Ni en Yacuy, ni en Metan existían escuelas agrarias previamente establecidas.

El estudio de base fue pensado como un diagnostico situacional que incluía aspectos demográficos, geográficos, económicos, y sociales de las 2 regiones pensadas para la ubicación de las escuelas EMETA. La inclusión de estas variables como eje de los diagnósticos en la provincia, puede pensarse a partir de la visión de los encargados en realizar el mismo, donde

3 Australes: moneda en curso creada durante el gobierno del presidente Raúl Alfonsín (1985). 
destacan principalmente ingenieros agrónomos, biólogos, economistas , arquitectos y en menor medida profesionales de las ciencias sociales (pedagogos, filósofos, historiadores), todos vinculados a la Universidad Nacional de Salta. De la matriz de conocimiento de estos profesionales es que puede suponerse el porqué de otorgar el acento a las cuestiones geográficas y económicas fundamentalmente, quedado relegado el relevamiento social a cuestiones más complementarias que centrales.

Para la elaboración de los informes se recolectaron fuentes de datos secundarios provenientes de Censos Nacionales de población y viviendas (1960, 1970 y 1980), Censo provincial de población aborigen (1983), Censo económico provincial (1985), Censo Nacional Agropecuario (1979). De hecho el informe diagnóstico de Metan (al igual que el de Yacuy) apelan constantemente a la transcripción de datos, cuadros y gráficos basado en estas datos secundarios.

Además se utilizaron registros de datos de: Atención Primaria de la Salud, Registros escolares del área EMETA- Yacuy, Informes departamentales elaborados por la secretaria de planeamiento de la provincia de Salta, Bioestadística de la provincia, Informe de la pobreza, Dirección de Minoridad de la Provincia de Salta, Informes Estadísticos y Cualitativos elaborados por los municipales de Metan y Tartagal.

\section{Diagnósticos para la implementación de las escuelas EMETA}

Para la zona de Metan, se tomaron como referencia datos relevados de localidades aledañas como lo son Anta y Rosario de la Frontera por ser departamento limítrofes con gran afluencia de sujetos que transitan de localidad en localidad, no solo por razones económicas (estas localidades están situadas sobre la ruta nacional $N^{\circ} 9$ que une Salta Capital con la Provincia de Tucumán), sino también por la cercanía entre las localidades (70 km de distancia entre las localidades) El informe diagnostico señala que:

- Anta y Metan presentan altos porcentajes de asalariados en el sector rural, $42,4 \%$ y 40,3 \% respectivamente. En Rosario de la Frontera aumenta "el cuenta propismo" y los familiares sin remuneración. 
-En zonas rurales, el porcentaje de estudiantes es en los 3 departamentos inferior a la media provincial. (ARGENTINA, 1988, p. 145)

-En las fincas se observan viviendas rurales tipo, de material donde habitan 2 o 3 familias de 4 o 5 chicos por matrimonio. No hay energía eléctrica y se utiliza farolas clásicas. (ARGENTINA, 1988, p. 164)

Es decir, se recabaron datos sobre cuestiones laborales, empleo, educación, condiciones de vida y vivienda. Los datos mostraban una alto índice del sector productivo en el sector rural, principalmente en las localidades de influencia, caracterizado socialmente por habitar casas precarias y con familia números, a la vez en el ámbito educativo el diagnostico mostraba un alto índice de deserción escolar principalmente en la escuela secundaria que superaba la media provincial.

En cuanto a las características de la tierra:

Se trata, en general de suelos profundos, medianamente bien provistos en materia orgánica, neutros, bien drenados, bien estructurados, con una buena disponibilidad de nutrientes minerales, desarrollados en terrenos relativamente altos, de pendientes suaves y con adecuadas y bien distribuidas precipitaciones como para asegurar las cosechas en la mayoría de los años. Estas tierras constituyen los sectores de mayor potencial agropecuaria (ARGENTINA, 1988, p. 31)

Algunas de las limitaciones de los suelos de estas tierras son: moderada susceptibilidad a la erosión hídrica, profundidad efectiva limitada moderada capacidad de retención de humedad, moderada probabilidad de sequias estacionales y ligeras deficiencias de drenaje (ARGENTINA, 1988, p. 32).

Estos factores adversos no se presentan conjuntamente y con la misma intensidad, por lo que si bien se descarta la práctica de cultivos de cosecha, muchos de estas tierras son aptas para la implementación de pasturas y otras sustentan pastizales naturales de variada capacidad forrajera (ARGENTINA, 1988, p. 33).

Hay que tener en cuenta que San José de Metan se encuentra ubicada en una cuenca rodeada de Sierras Subandinas y Llanura Chaqueña, lo que le da un clima subtropical serrano, donde las máximas precipitaciones 
se dan en verano (900 $\mathrm{mm}$ anuales aproximadamente), y donde las geográficas del lugar y las zonas aledañas son vegetación selvática perteneciente a las Yungas, con gran diversidad de flora y fauna.

Además Metan se caracteriza por ser un centro agrícola-ganadero activo en la provincia, destacándose la producción de oleaginosas: soja, porotos, cereales, ganadería bovina y frutales: cítricos principalmente.

Los estudios de base incluían también aspectos vinculados a la organización que debía seguir la escuela EMETA en la zona de Metan. La distribución de roles y la asignación de funciones para cada departamento de la estructura fue pensada para optimizar el funcionamiento de la institución, a la vez que el trabajo en conjunto de los distintos departamentos especializados en actividades específicas.

Se determinaron áreas de influencia en ambas escuelas en función de:

a) Mercado ocupación del egresado

Con respecto a la escuela de Metan el área de influencia en función del mercado ocupacional del egresado abarcaba, en el estudio los departamentos de Metan, Rosario de la Frontera y Anta, este último porque se ha constituido en el aérea natural de expansión de los productores de los 2 primeros departamentos.

Con respecto a la escuela de San José de Yacuy (Tartagal) se tomó la totalidad del departamento San Martin y el Departamento Oran desde Colonia Santa Rosa hacia el norte, con las localidades de Pichanal, Oran hasta la frontera con Bolivia en cuanto a cultivos intensivos bajo riesgo y el resto del departamento bajo cultivo extensivo

b) Área de influencia del alumnado ingresante

Con respecto a la escuela de Metan, el área de influencia del alumnado ingresante, se concentró en las poblaciones de Rio Piedras, Metan y Rosario de la Frontera y poblados intermedios como lugares de captación de alumnos para la escuela, no así de Joaquín V. González, que inicialmente se encontraba prevista, dado que no pueden asegurarse fehacientemente la concurrencia de alumnos de esta localidad debido a que los medios de transporte son menos asiduos y la carencia de albergue en la escuela limita el acceso de estos sectores, lo que no implica descartar 
que la institución absorba alumnos que tradicionalmente concurren a Metan, residiendo en hogares de parientes o pensiones, que no pueden ser cuantificados para este estudio (ARGENTINA, 1988, p. 2).

Con respecto a San José de Yacuy, el área de influencia del ingresante abarcaría desde el límite norte en la frontera con Bolivia, incluyendo las localidades ubicadas sobre la ruta 34 hasta Coronel Cornejo. (ARGENTINA, 1988, p. 3). De esta forma cada segmento de la organización tenía a cargo funciones específicas vinculantes con otro sector de la estructura:

Asamblea institucional: se reuniría 2 veces por año, al inicio de cada cuatrimestre. Esta asamblea estaba constituía por el consejo directivo presidida por el Director del establecimiento, con la finalidad de establecer acuerdos y tomas de decisiones que se considerasen necesarias para la gestión de la escuela. Puede leerse aquí, un intento no menor de democratización de las decisiones a nivel de gestión institucional. Consejo Directivo: compuesto por una Sala cooperativa y una sala académica que funcionan separadamente.

- Sala cooperativa: determinaría los lineamientos de producción y comercialización de los productos agropecuarios. Su función era la de coordinar con la sala académica los alcances de la educación formal y del centro de Educación Permanente en función de la demanda del mercado laboral y del desarrollo comunitario, además de compra de agroquímicos y fomentar la conciencia cooperativa. La misma estaría conformada por personal de la institución y familiares de los alumnos (padres).

- Sala académica: su misión era entender las políticas y vías de acción para el logro de los objetivos del proyecto educativo. Su función era pautar la gestión académica y atender las necesidades de apoyo material para el normal desarrollo del proceso de enseñanza- aprendizaje (ESTUDIO DE BASE II- Organización de las Unidades Ejecutivas, 1988, p. 20) Coordinador productivo: destinado a desarrollar actividades prácticas, aplicar régimen de trabajo y de disciplina a los trabajadores a su cargo, distribuía el personal de campo de la toda la unidad, intervenía en la calificación y cualificación del personal a cargo. Su misión era organizar y coordinar las tareas de campo (ESTUDIO DE BASE II- Organización 
de las Unidades Ejecutivas, 1988, p. 26-27).

Coordinación Pedagógica: Integraba la sala cooperativa y la sala académica y brindaba asesoramiento sobre: conducción institucional, programas y planificaciones, aspectos del proceso enseñanza- aprendizaje. Además organizaba y conducía las reuniones interáreas y tenía cargo la biblioteca y el laboratorio. Su misión era coordinar todas las acciones referentes a los procesos pedagógicos y socio educativos (ESTUDIO DE BASE IIOrganización de las Unidades Ejecutivas, 1988, p. 29-30).

Centro de Educación Permanente: pensado para brindar servicios educativos acorde a las demandas de las zonas. Buscaba actividades que implicasen una acción más extensiva y comprometida de modo que se revierta en la participación de la comunidad. Su finalidad era promover el desarrollo de las comunidades rurales. (ESTUDIO DE BASE II- Organización de las Unidades Ejecutivas, 1988, p. 33).

Ecónomo: Era el encargado del servicio de alimentación de los alumnos, el orden y la limpieza de los locales e instalaciones de Economato, dependencias escolares, laboratorios y dependencias administrativas. Su función era organizar y controlar lo referente a la provisión y régimen de alimentación de los alumnos (ESTUDIO DE BASE II- Organización de las Unidades Ejecutivas, 1988, p. 38).

La organización se mostraba compleja en comparación a la organización pensada para la localidad de Yacuy. Esta última no tenía en su estructura de gestión prevista un Consejo Directivo, solo Director, a la vez que suplantaba la Coordinación Productiva y la Pedagógica por un Consejo Consultivo Pedagógico- Cooperativo.

La estructura de Gestión de Escuela de Metan, planteaba en definitiva una subdivisión de tareas, pensando en la democratización de la decisiones a la vez que proveía a las instituciones de estamentos de autocontrol intra institucional, respondiendo además a una estructura con marcadas características empresariales de división de responsabilidades y actividades.

El 11 de Junio de 1990, en un predio de 90 hectáreas aproximadamente, en el kilómetro 1459 sobre la ruta Nacional $N^{\circ} 34$, se inaugura y entra en funcionamiento oficialmente la Escuela de Educación Técnica EMETA 
1 de la localidad de Metan Viejo- Salta. Su trayecto técnico profesional fue la formación en producción agropecuaria, pero sufrió los avatares propios de la restructuración del sistema educativo provincial a partir de las adaptaciones que se iniciaron en la provincia en 1995, con la ley de educación provincial $N^{\circ} 6829$

Con edificio construido especialmente bajo las normas establecidas por la Unidad Ejecutora Nacional, en un predio preparado para el funcionamiento de la escuela, y con un plan de estudio orientado a la formación agrícola-ganadera propia de la zona.

Estas variables constituyeron las bases de la planificación del programa para esta localidad, a partir de cada una de ellas se fueron trabajando tanto el currículum como las estrategias de implementación.

La escuela EMETA Yacuy (Aguaray) se creó en 1992 y comenzó funcionando en la escuela primaria de la misma localidad, hasta su inauguración en el año 1994, donde comenzó a funcionar en su actual edificio. Actualmente cuenta con 200 has de tierras, de las cuales aproximadamente 80 has se encuentran desmontadas.

El plantel educativo estuvo conformado por 42 profesionales y una matrícula de 270 alumnos. Su orientación desde mediados de los noventa fue la Modalidad de Bienes y servicios, con orientación en la producción Agropecuaria y con trayecto técnico profesional: Técnico en Instalaciones Agropecuarias.

\section{A modo de cierre: las transformaciones en los 90}

Entrado los 90, las reformas se caracterizaron fundamentalmente por cambios en los modelos de financiamiento, exigencia de eficiencia a través de la implantación de sistemas evaluativos, y presiones por relaciones más estrechas con el sector productivo (GARCÍA GUADILLA, 2008)

Esta década se caracterizó, por profundos cambios en el panorama internacional, modificando las condiciones sociales, económicas, culturales y políticas de gobierno del Estado, poniendo restricciones a sus políticas públicas y, en consecuencia, generando una fuerte conmoción en el sistema educativo, históricamente encargado de la función de 
reproducción cultural y socialización.

La Argentina no estuvo ajena a los procesos de las principales reformas impulsadas por el neoliberalismo norteamericano, que tendieron a subordinar la política educativa a la política económica fijada entre los países llamados "emergentes" y el Fondo Monetario Internacional.

El equipo ministerial y los expertos del Banco Mundial decidieron aplicar en la Argentina la Reforma española que, iniciada en los últimos años del Franquismo y con una posterior evaluación negativa de sus resultados, había sido derogada por el gobierno socialista de Felipe González.

Este periodo marca una etapa intensa en cuanto a la producción de nuevas bases legales y curriculares para el campo de la educación. Las políticas educativas adoptan una configuración legislativa a la par de la formulación de una reforma nacional y un programa estatal.

En 1991, con la Ley de Transferencias N²4.049, se disuelve el CONET. Poco tiempo después, en 1993 se sanciona la Ley Federal de Educación Nº 24.195, que en su estructuración deja de lado la modalidad técnica de nivel medio, y crea el nivel Polimodal de 3 años. Posteriormente, se transfieren las escuelas de educación técnica a las provincias y se agregan los llamados Trayectos Técnico Profesionales (TTP) para intentar compensar algunos de los contenidos específicos perdidos con la sanción de la ley.

Un análisis particular merece en este marco la Educación Técnico Profesional, modalidad olvidada en esta etapa. La nueva estructura educativa prescripta por la LFE, contempló una Educación General Básica (EGB) obligatoria de diez años, un ciclo Polimodal de tres, sin hacer referencia alguna a la Educación Técnica. Por otra parte, el contexto de quiebre de la industria nacional y de cambios en la estructura y volumen del empleo desdibujaron su rol. A pesar de que el "trabajo" no había aparecido en la historia de la educación argentina como eje principal del discurso educativo, el modelo organizacional de la Educación Técnica -previo a la reforma- había conformado durante muchos años un circuito destinado a garantizar el aporte de recursos humanos a la producción y los servicios. El nuevo escenario quitó sentido a esta modalidad educativa al caer un modelo de desarrollo en el que el trabajo había tenido un lugar destacado y sólo le permitió mantener parte de su espacio en algunos proyectos o iniciativas espontáneas desarrolladas por las instituciones escolares, o en planes y programas elaborados a partir de acuerdos establecidos 
por las provincias en el Consejo Federal de Cultura y Educación. (CORDERO; BUCCI, 2011, p. 161)

Esta ley traía aparejado en su cambio de estructura, la creación E.G.B. (Educación General Básica), con un período de nueve años de escolaridad obligatoria que sustituyó a los tradicionales siete años de la escuela primaria e incorporó los dos primeros años del viejo secundario. De esta forma se desarmó la estructura de la enseñanza media, reduciendo la misma a 3 años de nivel polimodal, y marcando como prioridad un tipo de formación generalista por encima de la especialización técnica o profesional.

Los contenidos de la escuela técnica se limitaron entonces a los últimos tres años del tradicional secundario, y con orientaciones previstas en los diseños curriculares del nivel polimodal, a saber: Producción de bienes y servicios; Economía y gestión de las organizaciones; Ciencias naturales; Humanidades y ciencias sociales, y Comunicación, artes y diseño.

Posteriormente, en 1996 se incorporaron los Trayectos Técnicos Profesionales (TTP), mediante acuerdos en el Consejo Federal de Educación como parte de la oferta educativa con la idea de sostener cierta forma de certificación profesional en áreas técnicas específicas.

A través del INET (Instituto Nacional de Educación Técnica), se perfilaron las bases curriculares de los TTP en diversas áreas: recreación y turismo; aeronáutica; salud y ambiente; construcciones-maestro mayor de obras; producción agropecuaria; construcciones-técnico; entre otras.

Las escuelas técnicas rurales no estuvieron al margen de estas transformaciones, quedando relegadas al ostracismo y al olvido en muchos casos. Las escuelas EMETA en la provincia de Salta siguieron funcionando a pesar del desfinanciamiento de la educación técnica, y lograron sostenerse en el tiempo, siendo ejes del desarrollo local en cada una de sus comunidades, siendo hasta el día de hoy centros educativos de referencias tanto en el norte, como al sur de la provincia. 


\section{Referencias}

AMADEO, T. La enseñanza y la experimentación agrícolas en la República Argentina. Dirección General de Enseñanza e investigaciones Agrícolas. Buenos Aires: Ministerio de Agricultura de la Nación, 1916.

ARGENTINA. Decreto n. 2.122, 29 de septiembre de 1987. Poder Ejecutivo de la Provincia de Salta.

AGENTINA. Ley 6.527, 1 de septiembre de 1988.Convenio de expansión y mejoramiento de la educación técnica agropecuaria. Poder Ejecutivo de la Provincia de Salta.

ARGENTINA. Ministerio de Educación y Justicia de la Nación. Banco Interamericano Desarrollo. Estudio de Base I - Diagnostico socioeconómico de las comunidades del proyecto y su área de influencia. Salta, 1988.

CORDERO, S.; BUCCI, I. Educación y mundo del trabajo. En busca de la recomposición del Sistema de Educación Técnica. Revista de Educación, Argentina, año 2, n. 3, p. 159-180, 2011.

ESTUDIO DE BASE II. Organización de las unidades ejecutivas. Ministerio de Educación y Cultura. Unidad Ejecutora Provincial. Programa EMETA. Salta, 1988

GARCÍA GUADILLA, C. Balance de la década de los 90 y reflexiones sobre las nuevas fuerzas de cambio en la educación superior. En. MOLLIS, M. Las Universidades en América latina: ¿reformadas o alteradas? La cosmética del poder financiero, Buenos Aires: Clacso, 2008

GUTIÉRREZ, T. Educación, agro y sociedad: políticas agrarias en 
la región pampeana, 1897-1955. 1ra Edición. Bernal: Universidad Nacional de Quilmes, 2007.

LEGUIZAMON, L.; JORGE NAVARRO, M. Escuelas EMETA en el norte Argentino. Estudio de Casos en La Rioja y Salta. X Jornadas Nacionales y II Internacionales de Investigación y Debate: II Encuentro Sudamericano de Estudios Agrarios. Actores, Estrategias y Poder en el Mundo Rural: América del Sur, 1850-2010. Buenos Aires: Mnemosyne, 2013.

MARTÍNEZ GUARINO, R. La escuela productiva. Utopía y realidad. Buenos Aires: Paidós, 1994.

DEPARTAMENTO DE ASUNTOS CULTURALES. La enseñanza agrícola en la Escuela Primaria. División Educación. Washington DC: Unión Panamericana, 1955.

SALVADORES, A. Rivadavia precursor de la enseñanza Agrícola en el país. Revista Humanidades. Tomo XXVIII: Historia y Geografía. Facultad de Humanidades y Ciencias de la Educación, 1940.

STAGNARO, J. M. Formación y perfeccionamiento del maestro de la escuela rural. En: JORNADAS PEDAGÓGICAS PANAMERICANAS, Buenos Aires, 6 al 13 de noviembre (Cap. III, Inciso c). Consejo Nacional de Educación. Auspiciadas por la Comisión Nacional Ejecutiva de homenaje al Sesquicentenario de la Revolución de Mayo, 1960.

VERGARA, C. N. La educación del niño y su instrucción por Víctor Mercante. La Educación, Argentina, p. 292-293, 1897.

Recebido: 03/07/2013

Aprovado: 16/12/2013 